J Neuropsychiatry Clin Neurosci. 2013 ; 25(4): 272-282. doi:10.1176/appi.neuropsych.12040078.

\title{
Psychiatric Disorders in Children and Adolescents Six-to-Twelve Months After Mild Traumatic Brain Injury
}

\author{
Jeffrey E. Max, M.B.B.Ch, David Pardo, B.S., Gerri Hanten, Ph.D., Russell J. Schachar, M.D., \\ Ann E. Saunders, M.D., Linda Ewing-Cobbs, Ph.D., Sandra B. Chapman, Ph.D., Maureen \\ Dennis, Ph.D., Elisabeth A. Wilde, Ph.D., Erin D. Bigler, Ph.D., Wesley K. Thompson, Ph.D., \\ Tony T. Yang, M.D., Ph.D., and Harvey S. Levin, Ph.D. \\ Dept. of Psychiatry, University of California, San Diego, and Rady Children's Hospital, San Diego, \\ CA
}

\section{Abstract}

The objective of this study was to understand how novel psychiatric disorders (NPD) in children with mild traumatic brain injury (MTBI) are related to pre-injury variables, injury-related variables, and concurrent neurocognitive outcome. A group of 79 children, ages 5 to 14 years, who had experienced MTBI, were studied from consecutive hospital admissions with semistructured psychiatric interviews soon after injury (baseline); 60 children were reassessed 12 months postinjury. Standardized instruments were used to assess injury severity; lesion characteristics; preinjury variables, including psychiatric disorder, family psychiatric history, family functioning, socioeconomic status, psychosocial adversity, adaptive functioning, and post-injury neurocognitive and adaptive functioning. NPD occurred in 17 of 60 participants (28\%) in the 6-12-month interval after injury, with disorders that were significantly associated with socioeconomic status, psychosocial adversity, estimated pre-injury academic functioning, and concurrent deficits in adaptive functioning, academic performance, processing speed, memory, and expressive language. NPD was not significantly associated with pre-injury adaptive functioning, injury severity, family psychiatric history, pre-injury psychiatric disorder, lesion location, gender, or age at injury. These findings suggest that the short-term psychiatric morbidity associated with MTBI in children occurs more commonly than previously reported and is related to both pre-injury social factors and concurrent neurocognitive functioning.

Of the 1.5 million traumatic brain injuries occurring annually in the United States, approximately 500,000 result in emergency department visits for children under 15 years of age, and $80 \% 290 \%$ of these cases are considered mild. ${ }^{1}$ Even if a small proportion of mild traumatic brain injury (MTBI) incidents in children result in behavioral problems, the large number of children affected represents a major public health concern. It is important, therefore, to consider whether MTBI is associated with new-onset or novel psychiatric disorder (NPD). A clear answer to this question has been hampered by a number of factors, including the lack of prospective (rather than retrospective) investigations, a large sample

Send correspondence to Dr. Max; jmax @ucsd.edu.

Disclosures: Dr. Schachar is a consultant for Eli Lilly Corporation and Purdue Pharma (Canada). None of the other authors has financial disclosures to make relative to for-profit enterprises. 
size sufficient to explore a range of injury and social variables, and a persisting assumption based on earlier investigations, that new psychiatric disorder after mild TBI may be unrelated to the brain injury per se. ${ }^{2} \mathrm{~A}$ better understanding of the genesis of NPDs in TBI would allow them to be predicted and perhaps treated early so as to limit morbidity.

NPD, by definition, can manifest in two ways. ${ }^{3}$ First, it could emerge after TBI in an individual with no pre-injury lifetime psychiatric disorder. Second, it could represent a novel disorder after TBI in an individual with another form of pre-injury lifetime psychiatric disorder (e.g., a patient with a pre-injury lifetime history of attention-deficit/hyperactivity disorder (ADHD) who develops a major depressive disorder after the TBI). To date, two prospectively-studied cohorts have been used to examine the relationship between MTBI and NPD. ${ }^{4}$ We found that children with mild/moderate TBI and a history of psychiatric disorder before the injury were at significantly higher risk for developing NPD in the first 3 months post-injury. ${ }^{3}$ Furthermore, after mild/moderate TBI, NPD occurred at rates of $8 / 37$ (22\%), 3/30 (10\%), 7/ $31(23 \%)$, and 6/30 (20\%), at assessments 3, 6, 12, and 24 months post-injury, respectively. ${ }^{4}$ We recently reported that NPD in the first 6 months after injury occurred in 25/70 (36\%) of children consecutively hospitalized for MTBI and that NPD was significantly associated with the presence of frontal white matter lesions. ${ }^{5}$

Additional investigations focused on specific symptom ratings, such as post-concussion symptoms (PCS), attention, and conduct, documented typically from brief parent and child interviews and/or questionnaires administered to parents, children, and teachers, as contrasted with psychiatric disorders derived from standardized psychiatric interviews. ${ }^{6-12}$ Results compatible with our findings ${ }^{3}$ were obtained in an earlier study of PCS, where children with MTBI whose symptoms increased had poorer pre-injury behavioral adjustment than those whose symptoms did not increase. ${ }^{11}$ Another study found that significant ongoing behavioral difficulty 3 months after injury in children with MTBI was related to higher incidence of previous TBI, premorbid stressors, pre-existing psychiatric or neurological problems, and learning difficulties. ${ }^{9}$ Findings from a recently-studied cohort of children with MTBI extended these PCS-related findings. $.7,10,12$ For example, a high acute level of PCS was likely among children with MTBI whose acute clinical presentation reflected more severe injury. ${ }^{10}$ PCS was significantly higher in an MTBI group than in an orthopedic injury (OI) control group 2 weeks after injury (51\% versus 30\%), but not thereafter in the first year post-injury (19\%227\% versus $19 \% 221 \%) .{ }^{12}$ Results from a birth cohort study indicated that MTBI resulting in inpatient, rather than outpatient, treatment was associated with increased ratings of hyperactivity/inattention and conduct disorder, especially if the MTBI occurred before age $5 .{ }^{8}$ Additional prospective ${ }^{13}$ and retrospective ${ }^{14}$ studies suggest varying neurobehavioral morbidity after MTBI.

In addition to behavioral and emotional morbidity that characterizes NPD, neurocognitive sequelae of TBI are important clinically; ${ }^{15}$ yet the relationship between NPD and neurocognitive deficits in an MTBI population is largely unstudied. Narrowing this knowledge gap is critical because it is clinically relevant to address pre-injury or post-injury general and specific cognitive deficits if they are present, especially within the context of NPD. We reported that NPD was significantly associated with concurrent deficits in processing speed, expressive language, and intellectual functioning 6 months after MTBI. ${ }^{5}$ 
We have found that NPD is significantly related to neurocognitive outcome in hospitalized children with a broader range of TBI severity (severe TBI and MTBI), and OI. ${ }^{16}$ Specifically, intellectual functioning and memory outcome were independently significantly related to a "neuropsychiatric factor" (encompassing severity of injury and NPD) and a "psychosocial disadvantage factor" (including socioeconomic status, family psychiatric history, and family functioning). NPD added significantly to severity of injury indices, and both family functioning and family psychiatric history added significantly to socioeconomic status (SES) in explaining several specific cognitive outcomes.

Aside from the few studies investigating the relationship of MTBI, NPD, and neurocognitive outcome, there are several other reports of MTBI, behavioral symptomatology, and neurocognitive outcome. One study found that children with MTBI whose "postconcussive symptoms" increased versus those whose did not increase from before injury to 3 months post-injury, performed significantly more poorly on tests of processing speed, visual memory, attention, and executive functioning. ${ }^{11}$ Another study found that children with MTBI and ongoing behavioral difficulties were not differentiated by their performance on verbal memory, visual memory, processing speed, attention, or executive function tests. ${ }^{9} \mathrm{~A}$ recent study found that measures of executive function were not significantly poorer in an MTBI versus OI group in the first year after injury. ${ }^{7}$ These negative findings are consistent with a recent study and reviews suggesting benign cognitive outcomes after MTBI. ${ }^{17-19}$ However, MTBI was more likely to result in PCS than was OI, among children of lower versus higher cognitive ability. This was especially evident for children with complicated MTBI (lesion detected on MRI). ${ }^{6}$

It is clear that children with MTBI do not necessarily escape psychiatric morbidity and that this morbidity may be related to neurocognitive morbidity. In this article, we explore each of these forms of morbidity in a large MTBI cohort studied prospectively and, in addition, investigate the relations between NPD and concurrent neuropsychological functioning. Considering the literature reviewed, we hypothesized that NPD in the 6-12-month postinjury interval in children with MTBI would be predicted by indices of injury severity (e.g., presence of a lesion; frontal white matter lesion), estimate of pre-injury academic functioning, and indices of psychosocial adversity (e.g., socioeconomic status, family psychiatric history, pre-injury family functioning). We further hypothesized that NPD (a broad category of psychopathology) at 12 months would be associated with significant deficits in concurrent adaptive and academic functioning, and a broad range (implying nonspecific rather than specific association) of neurocognitive measures, including processing speed, memory, and expressive language.

\section{METHODS}

\section{Participants}

Participants were recruited from three academic medical centers in Texas $(\mathrm{N}=60)$, and The Hospital for Sick Children in Toronto ( $\mathrm{N}=19)$ following consecutive admissions during their initial hospitalization for MTBI from July 1998 to August 2002. Children recruited from an additional site, in San Diego, were excluded because of some differences in inclusion/ exclusion criteria. This exclusion did not alter the results of the study in any meaningful 
way. Children were considered to have sustained an MTBI if their injury was via a closed head injury mechanism, lowest Glasgow Coma Scale score (GCS $\$ 13)^{20}$ on examination in the emergency center, and a history of an altered or loss of consciousness not exceeding 30 minutes. ${ }^{21}$ Consistent with the leading neurobehavioral study on pediatric MTBI, children were not excluded if they suffered a skull fracture. ${ }^{10}$ We do not have accurate data on the eligibility or number of children approached or participation rate among those eligible. This is partly a result of not requiring our patients to answer eligibility questions before making a decision on whether to participate. Exclusion criteria included injuries due to child abuse or penetrating missiles, mental deficiency, pre-existing schizophrenia, or autistic spectrum disorder. Informed consent was obtained from the parents/guardians of all child participants. The children all provided assent to participate in accordance with the Institutional Review Board requirements at each site. A group of 79 children enrolled and were studied 2 weeks after injury. One child had a second TBI between the 6- and 12-month follow-up assessments and was excluded from the analyses; 60 children (76\%) returned 12 months post-injury for reevaluation. The returning group was not different from the non-returning group with respect to GCS scores, age, gender, race, SES, pre-injury lifetime psychiatric disorder, pre-injury family functioning, pre-injury family psychiatric history, or pre-injury adaptive functioning. However, the returning group had significantly higher psychosocial adversity (mean [standard deviation]: $0.75(0.90), \mathrm{N}=59$ versus $0.39(0.50), \mathrm{N}=18 ; t[52.2]=$ 22.14; $\mathrm{p}=0.037)$.

Table 1 presents data on demographics (age, gender, SES), pre-injury psychosocial variables (pre-injury lifetime psychiatric status, adaptive functioning, family functioning, family psychiatric history, psychosocial adversity), and injury indices (GCS scores, depressed skull fracture incidence, mechanism of injury). Race of participants was as follows: Caucasians: 51 (65\%); African-American: 12 (15\%); Hispanic: 12 (15\%); Asian: 2 (3\%); Other: 2 (3\%).

\section{Measures}

Psychiatric Assessment-A semistructured interview, the Schedule for Affective Disorders and Schizophrenia for School-Aged Children, Present and Lifetime Version (KSADS-PL), ${ }^{22}$ was used to establish DSM-IV psychiatric diagnoses. ${ }^{23}$ The K-SADS-PL is an integrated parent- child interview that involves the synthesizing of data collected by a clinician from parent and child separately, including present and lifetime symptoms (at baseline) and symptoms present or past from 6-months post-injury to 12 months (12-month assessment). We also administered a semistructured interview designed to identify symptoms and subtypes of the DSM-IV diagnosis of Personality Change Due to TBI, called the Neuropsychiatric Rating Schedule (NPRS). ${ }^{24}$ Both parents and children participated in the interview, which occurred at baseline and at 12 months post-injury.

The reports of the parent and child from the NPRS, K-SADS interviews, and the Survey Diagnostic Instrument, ${ }^{25}$ completed by the teacher when available (51/79: 65\%, at baseline; 40/60: 67\%, at 12 months), were integrated. The interviewer generated "best-estimate" psychiatric diagnoses, considered the "gold standard" of child psychiatric assessment, integrating data from multiple sources. ${ }^{26}$ 
Interviews were performed by Master's- and Ph.D.-level clinicians who were trained by the first author in a pre-study workshop and a mid-study workshop. The assessments at each location were supervised by a child psychiatrist (at three sites) or a child psychologist (at one site). The first author was responsible for a second level of supervision, which involved reviewing written summaries composed by the interviewer and discussion of cases at monthly teleconferences between the first author and the interviewers.

Neurological Assessments-Severity of TBI was determined from the lowest score on the Glasgow Coma Scale (GCS $)^{20}$ recorded from clinical notes. The GCS has been used as a standard measure of severity of acute brain injury associated with TBI. The scale measures eye-opening and verbal and motor responsiveness out-putting scores that range from 3 (unresponsive) to 15 (normal).

The Abbreviated Injury Scale (AIS) was used to measure overall extracranial injury severity and provide an Injury Severity Score (ISS). ${ }^{27}$ The ISS was calculated from the sum of the squares of the highest AIS score in each of the three most severely injured body regions (chest, abdominal or pelvic contents, extremities, and external), if applicable.

Magnetic Resonance Imaging (MRI, 1.5 Tesla) was conducted in the majority of participants 3 months after the injury, when lesions appear stable. Included in the protocol were fluid attenuated-inversion recovery (FLAIR) sequences and $\mathrm{T}_{1}$-weighted volumetric spoiled gradient recalled echo (SPGR), acquired in coronal and sagittal planes according to a research protocol. Coding of lesions included gray/white matter pathology (e.g., shearing injury, gliosis, hemosiderin) and anatomical location. Frontal-lobe white-matter lesions were described as present or absent. Specific coding of frontal-lobe gyri was made only when gray-matter lesions appeared in these gyri. There was no attempt to register images or to segment tissue types because the coding of lesions was done by expert project neuroradiologists at each site, and volumetric analyses were not conducted; 65 of the 79 enrolled and eligible children (82\%) completed their research MRI. The lesion distributions in the children who completed the MRI are displayed in Table 2. Ten of the children with missing 12-month psychiatric data had a research MRI. Lesion presence and specific location on the research MRI did not differ in those with psychiatric follow-up versus those without.

\section{Psychosocial Assessments-The Family History Research Diagnostic Criteria ${ }^{28}$} interview was administered at each site by trained research assistants. The criteria were changed to conform to DSM-IV criteria. At least one parent, acting as the informant, was questioned about psychiatric disorders in each first-degree relative of the index child with a TBI. Using a 4-point scale of increasing severity, the family ratings were then summarized. ${ }^{4}$

Global family functioning was measured with the Family Assessment Device General Functioning Scale. ${ }^{29}$ The scale is a self-report questionnaire consisting of 12 items. The primary caretaker in each family answered each question on a 4-point Likert scale ranging from 1-4. Higher scores represent greater dysfunction. 
The Four-Factor Index was used to assess socioeconomic status (SES). ${ }^{30}$ Classification is based on scores derived from a formula involving both the paternal and maternal educational and occupational levels. Higher scores indicate higher SES, ranging from 8 to 66 .

We modeled our psychosocial adversity index after that used in an important early study of pediatric TBI. ${ }^{2}$ The assessment involved six areas, and, for each area that suggested adversity, a score of 1 was given; for each area where there was no adversity, a score of 0 was given. The areas are 1) child not living with biological or adoptive parents; 2) sibship of at least 4 children or a person:room ratio exceeding $1 ; 3$ ) admission of the child into the care of local authorities because of family difficulties; 4) maternal "malaise inventory" score of $\$ 7 ; 5$ ) paternal criminality; and 6) father or mother with an unskilled or semi-skilled job.

Pre-injury adaptive functioning was retrospectively assessed within 2 weeks after the injury, and adaptive functioning at 12 months post-injury was assessed with the Vineland Adaptive Behavior Scale interview, ${ }^{31}$ which involved a semistructured interview conducted by a trained research assistant with the primary caretaker.

Neurocognitive Assessments: Estimate of Pre-injury Academic FunctioningAcademic functioning at baseline (within 2 weeks of injury) was assessed with the Woodcock-Johnson Revised Letter-Word Identification subtest. ${ }^{32}$ The Letter-Word Identification assesses the accuracy of reading letters and words aloud. A standard score was generated representing the total number of items read correctly. Other studies have presented evidence that, in children with mild TBI, the baseline post-injury assessment of this academic functioning domain can be used to estimate pre-injury academic functioning, ${ }^{33}$ although pre-injury academic functioning depends on other factors, as well.

\section{Concurrent Academic and Neurocognitive (Processing Speed, Memory, Language) Functioning (12 months post-injury)}

Academic Functioning-Academic functioning at 12 months was assessed with the Woodcock-Johnson Revised Letter-Word Identification subtest ${ }^{32}$ described above.

Processing Speed-The Wechsler Intelligence Scale for Children III (WISC-III) Coding and Symbol Search subtests were used to measure processing speed. ${ }^{34}$ During the coding subtest, children identified the correct geometric designs below numbers by use of a key. The test measured the number of symbols correctly transcribed in 2 minutes. The Symbol Search subtest involved presenting the child with target stimuli and being asked to check a Yes or No box as fast as possible to indicate whether or not the target(s) appeared among an array of stimuli ( 45 total trials). The score was derived by subtracting the number of errors from the number of correct responses made in 120 seconds. A Processing Speed scaled score was calculated and averaged for both subtests.

The Rapid Automatized Naming task ${ }^{35}$ was administered by asking the child to rapidly name line-drawings of five common objects reproduced 10 times each and interspersed on a board. This task is related to processing speed and reading. The time required to complete the task was the dependent measure and was expressed as a z-score. 
Memory-The California Verbal Learning Test-Children's Version (CVLT-C) was administered as an assessment of children's verbal learning memory abilities. ${ }^{36}$ The CVLT$\mathrm{C}$ standard procedures for alternate forms were followed. Children were instructed to learn 15 words in 3 categories across 5 learning trials and 1 distraction trial. Verbal memory was assessed for Delayed Recall and was documented as a z-score.

Working memory was tested with an N-back task presented in central vision by a Macintosh Powerbook computer with varying memory load. ${ }^{37} \mathrm{~A}$ 0-back condition imposed a minimal memory load while controlling for attention-to-task. At each level of memory load, there were 40 trials, in which the child viewed a continuous string of 40 letters appearing 1 letter at a time on the computer screen for 2 seconds each, onset-to-onset. Within each level of the task, there were 12 targets and 28 distracters. The child responded by pressing a button with the preferred hand when a match occurred, or, in the 0-load condition, when a designated target appeared. The percentage of hits (i.e., detection of targets) for the Letter Rhyme task were recorded.

Language-The Clinical Evaluation of Language Fundamentals, 3rd Edition (CELF23) Formulated Sentence subtest was used to assess expressive language at the sentence level. ${ }^{38}$ The test involved presenting the children a visual picture that contained a target word or phrase and asking them to formulate one sentence in response. The subtest contained 22 items.

\section{Data Analysis}

Statistical tests were independent-sample $t$-tests or $\chi^{2}$ analyses and effect-size analyses ${ }^{39}$ as appropriate. Alpha levels were set at 0.05 . We tested the association of 12-month post-injury NPD with injury and pre-injury variables of interest (presence of lesion, frontal white matter lesion, SES, psychosocial adversity, pre-injury family functioning, family psychiatric history). We analyzed the relationship of NPD with baseline academic testing scores (Woodcock-Johnson-R Letter-Word ID scale) as well as concurrent neurocognitive functioning 12 months post-injury for specific domains sensitive to disruption after MTBI (academic functioning, processing speed, memory, language). We also conducted exploratory analyses of variables potentially associated with NPD, including demographics (age at injury, gender, race), pre-injury psychosocial (pre-injury lifetime psychiatric status, adaptive functioning), and injury (GCS scores, depressed skull fracture) variables.

\section{RESULTS}

Pre-Injury and Novel Psychiatric Disorders (NPD) In the group, 31 of the enrolled children $(31 / 79 ; 39 \%)$ had a history of at least one lifetime pre-injury psychiatric disorder.

Specifically, these disorders included ADHD $(\mathrm{N}=20)$; simple phobia $(\mathrm{N}=7$, including 2 in remission); separation anxiety disorder ( $\mathrm{N}=5$, including 2 in remission); oppositional defiant disorder ( $\mathrm{N}=3$, including 1 in remission); generalized anxiety disorder $(\mathrm{N}=2)$; obsessivecompulsive disorder $(\mathrm{N}=1)$; encopresis $(\mathrm{N}=1)$; disruptive behavior disorder, not otherwise specified $(\mathrm{N}=1)$; eating disorder, not otherwise specified $(\mathrm{N}=1)$; social phobia $(\mathrm{N}=1)$; chronic motor tic disorder $(\mathrm{N}=1)$; and major depressive disorder $(\mathrm{N}=1$, in remission). 
NPD occurred in $28 \%$ of the children who returned for the 12-month assessment (17/60). The specific disorders included ADHD $(\mathrm{N}=6)$, adjustment disorder $(\mathrm{N}=2)$, social phobia $(\mathrm{N}=1)$, simple phobia $(\mathrm{N}=2)$, generalized anxiety disorder $(\mathrm{N}=3)$, motor tic disorder $(\mathrm{N}=2)$, oppositional defiant disorder $(\mathrm{N}=3)$, personality change $(\mathrm{N}=4)$, major depressive disorder $(\mathrm{N}=2)$, posttraumatic stress disorder $(\mathrm{N}=2)$, and separation anxiety disorder $(\mathrm{N}=1)$.

\section{Pre-Injury and Injury Correlates of Novel Psychiatric Disorder (NPD)}

Table 3 and Table 4 present the results of the analyses conducted to determine which variables were significantly associated with development of NPD 6-12 months after TBI. As hypothesized, SES, psychosocial adversity, and estimated pre-injury academic functioning showed significance. Lower pre-injury family functioning tended to be associated with NPD, and the moderate effect size suggested insufficient power. None of the other demographic or psychosocial variables (age at injury, gender, race, family psychiatric history, pre-injury adaptive functioning, or pre-injury psychiatric disorder) was associated with NPD, nor were injury characteristics such as lowest GCS score, depressed skull fracture, abnormality detected on CT scan, or extracranial injury severity.

\section{NPD at 12-Month Assessment: Neurocognitive and Adaptive Functioning Correlates at 12 Months}

Table 4 presents the results of the neurocognitive and adaptive functioning correlates at 12 months. The assessments of academic functioning (W-J-R Letter-Word ID), processing speed (Rapid Naming), memory (N-back Letter Rhyme task; CVLT-C), expressive language (CELF23), and adaptive functioning showed significance in independent-sample $t$-tests. Exploratory analyses demonstrated that the adaptive-functioning results were accounted for by all domains (socialization, communication, daily living skills). The significant relationship between 12-month adaptive functioning and NPD was maintained also in a logistic regression controlling for pre-injury adaptive functioning.

In view of the significant association of SES and NPD, we repeated the analyses of the relationship of NPD and its significantly associated neurocognitive measures, controlling for SES in a series of logistic regressions. The regressions were significant, but there was no instance where SES independently significantly accounted for NPD. Working memory (Nback Rhyme task) and verbal memory (CVLT-C) were the only neurocognitive measures that accounted for significant variance independent of SES.

\section{Lesion Characteristics}

The lesion distributions from the MRI research are listed in Table 2. The presence or absence of lesions was not significantly associated with NPD (lesions were present in 7/15 children with NPD versus 20/40 children with no NPD). Furthermore, the presence or absence of frontal white-matter lesions was not significantly associated with NPD (frontal white-matter lesions were present in 2/15 children with NPD versus $3 / 40$ children with no NPD). 


\section{DISCUSSION}

The main findings of this study are that novel psychiatric disorders (NPDs) 6-12 months after mild traumatic brain injury (MTBI) are common, are associated with significant deficits in adaptive functioning, and are significantly associated with pre-injury psychosocial risk factors, including lower SES and psychosocial adversity.

Our findings also suggest that NPD is significantly related to estimated pre-injury academic functioning. Finally, NPD appears to be associated with deficits in concurrent neuropsychological functioning across multiple cognitive and academic domains.

NPD after MTBI occurred at a relatively high rate, appearing in 28\% (17/60) of the children who returned for the 12-month assessment. This rate is slightly higher than the rate reported by a previous study, which found that 7 of 31 children and adolescents (23\%) expressed NPD 6-12 months after mild and moderate brain injuries. ${ }^{40}$ Either result has significant public health implications, because of the high incidence of MTBI. ${ }^{1}$ The specific NPDs expressed by the children were heterogeneous, a result similar to those of other pediatric TBI studies. ${ }^{2,40}$ The most frequently occurring NPDs were secondary ADHD and personality change due to TBI, followed by mood, anxiety, and behavioral disorders. The secondary ADHD finding is particularly striking because 25\% (20/79) of the participants enrolled at baseline had pre-injury ADHD and were thus not eligible to develop secondary ADHD. The high rate of pre-injury ADHD, a two-to-three-fold increase, as compared with community prevalence, is consistent with other studies of pediatric TBI and may be related to impulsivity as a contributing causal factor to injury. ${ }^{41}$

NPD was significantly associated with SES and psychosocial adversity. NPD was also clearly related to other pre-injury psychosocial measures, including family psychiatric history and family functioning, with small-to-moderate effect sizes, respectively. These results contrast with those of the same cohort with respect to NPD in the first 6 months after injury, in that the significant association with frontal lobe white-matter damage is no longer apparent. ${ }^{5}$ As time-since-injury increases, the relationship of brain injury indices and psychiatric outcome may decrease, ${ }^{2,42,43}$ and there may be a closer connection with psychosocial factors that influence daily life. This pattern is by no means uniform, such that the reverse may be true (e.g., regarding oppositional defiant disorder symptomatology) ${ }^{44}$ or changing injury, and psychosocial correlates may occur with the passage of time (e.g., in the case of personality change due to TBI) ${ }^{45,46}$ However, the association between psychosocial adversity and NPD/neurobehavioral deficits is one of the most consistent findings across cohorts internationally in pediatric TBI research. , $^{2,41,47-49}$

Estimated pre-injury academic functioning was significantly related to NPD. Not surprisingly, estimated pre-injury academic functioning was related to SES ( $\mathrm{r}=0.377$; $\mathrm{p}=0.004$ ). Together, these findings suggest that children with pre-injury psychosocial adversity and associated pre-injury lower academic functioning are at increased risk for the development of NPD. The fact that authoritative reviews ${ }^{18,19}$ and a recently published study ${ }^{17}$ find benign neurocognitive outcome after pediatric MTBI, makes it unlikely that the early post-injury deficits in academic functioning associated with NPD in this study 
represent a decline in functioning or are due to brain damage. However, the latter-referenced study had participants with milder injuries than those described in this investigation.

Neurocognitive deficits present 12 months post-injury were associated with NPD across multiple domains of functioning with small-to-large effect sizes. Neurocognitive functioning was so closely tied to SES that an independent relationship of neurocognitive outcome (except for memory function) or SES to NPD could not be consistently demonstrated in regression analyses. This suggests that the neurocognitive deficits were already present before the MTBI in the children who went on to develop NPD. Therefore, children with neurocognitive deficits or lower neurocognitive reserve ${ }^{50}$ may be at increased risk for NPD or other behavioral problems. ${ }^{5}$ Nevertheless, it remains at least possible that the MTBI caused the significant neurocognitive deficits.

In contrast to the-at least possible-argument about the sequential connection between MTBI and neurocognitive deficits, the methods of this psychiatric study allowed confident documentation of new psychopathology (i.e., NPD, after MTBI). The children with NPD at 12 months post-injury had significantly lower adaptive functioning than counterparts with no NPD. Interestingly, this relationship remained significant even when pre-injury adaptive functioning was controlled. There are several implications of this finding. First, the construct of NPD is clinically meaningful and relates not simply to psychopathology but also to adaptive dysfunction. Second, behavioral change in the form of a psychiatric disorder clearly occurs after MTBI and is associated with a decline in adaptive functioning. These findings, in juxtaposition to the only possible likelihood of significant changes in neurocognitive functioning after MTBI, suggest that NPD may be a more sensitive index for the effects of MTBI. However, an injured control group (e.g., with OI and no TBI), might clarify to what extent NPD at 12 months post-injury may be due to brain damage per se.

The results of this study must be considered within its limitations. First, the MTBI sample was limited to hospitalized children, and there is a growing trend for children with MTBI to be discharged from emergency rooms. ${ }^{51}$ Thus, our sample may differ from the entire population of children who have experienced MTBI. As a result, the sample may also reflect negative injury or psychosocial factors that would influence whether the child is hospitalized. An example of a negative injury factor is the high rate of children with an abnormal MRI (48\%), compared with a cohort recruited regardless of hospitalization status $(17 \%) .{ }^{10}$ Second, interrater reliability assessments for the diagnosis of NPD were not directly evaluated on the basis of videotaped interviews. However, the assessments at each site were closely supervised by the child psychiatrists or psychologist. Furthermore, frequent telephone conferences were held, and transmission of written psychiatric assessment summaries were critiqued by the first author and the other interviewers, which helped maintain fidelity in diagnosis across sites. Third, attrition in terms of participation of enrolled and eligible children was $24 \%$, although there were no differences in injury, demographic, or psychosocial variables between the children who returned and those who did not, except for lower psychosocial adversity in children who did not return. Thus, the $28 \%$ rate of NPD may be an overestimate. However, even if none of the participants lost to attrition developed an NPD, the rate would still be high $(17 / 79 ; 22 \%)$. Fourth, the image analysis did not use volumetric measurements or diffusion tensor imaging, which could have 
more clearly delineated imaging correlates of NPD. Fifth, although our hypotheses did not include an orthopedic injury (OI) comparison group, such a group could serve as a control for NPD in children predisposed to and exposed to injuries.

The strengths of this study should also be appreciated. This is the largest prospective psychiatric interview study of a consecutively-hospitalized population of pediatric MTBI. The breadth and depth of assessments were extensive and included interview assessments of psychopathology, adaptive functioning, and family psychiatric history, in addition to rating scales representing injury and other psychosocial risk factors for NPD, as well as assessments of several domains of neurocognitive functioning. Furthermore, expert neuroradiologists performed the lesion analysis.

\section{CONCLUSIONS AND IMPLICATIONS}

Children who experience MTBI should continue to be monitored and screened for the development of NPD within the first 12 months after injury. Individuals with marginal preinjury academic and neurocognitive functioning, lower SES, greater psychosocial adversity, or those who show a decline in school performance should be monitored particularly carefully. We are currently conducting an urgently-needed large, controlled prospective psychiatric study of consecutively-treated children with MTBI regardless of emergency room disposition to inhospital treatment or discharge. The controls are age- and SESmatched children with OI, which allows a determination of the extent of the psychiatric morbidity related to this very common type of TBI.

\section{Acknowledgments}

This study was supported by National Institute of Mental Health (NIMH) Grant K-08 MH01800 (Dr. Max) and National Institute of Neurological Disorders and Stroke (NINDS) Grant NS-21889 (Dr. Levin).

\section{References}

1. Bazarian JJ, McClung J, Shah MN, et al. Mild traumatic brain injury in the United States, 19982000. Brain Inj. 2005; 19:85-91. [PubMed: 15841752]

2. Brown G, Chadwick O, Shaffer D, et al. A prospective study of children with head injuries, III: psychiatric sequelae. Psychol Med. 1981; 11:63-78. [PubMed: 7208747]

3. Max JE, Smith WL Jr, Sato Y, et al. Traumatic brain injury in children and adolescents: psychiatric disorders in the first three months. J Am Acad Child Adolesc Psychiatry. 1997; 36:94-102. [PubMed: 9000786]

4. Max JE, Robin DA, Lindgren SD, et al. Traumatic brain injury in children and adolescents: psychiatric disorders at two years. J Am Acad Child Adolesc Psychiatry. 1997; 36:1278-1285.

5. Max JE, Schachar RJ, Landis J, et al. Psychiatric disorders in children and adolescents in the first six months after mild traumatic brain injury. J Neuropsychiatry Clin Neurosci. 2013; 25:187-197.

6. Fay TB, Yeates KO, Taylor HG, et al. Cognitive reserve as a moderator of postconcussive symptoms in children with complicated and uncomplicated mild traumatic brain injury. J Int Neuropsychol Soc. 2010; 16:94-105. [PubMed: 19835663]

7. Maillard-Wermelinger A, Yeates KO, Gerry Taylor H, et al. Mild traumatic brain injury and executive functions in school-aged children. Dev Neurorehabil. 2009; 12:330-341. [PubMed: 20477562] 
8. McKinlay A, Dalrymple-Alford JC, Horwood LJ, et al. Long term psychosocial outcomes after mild head injury in early childhood. J Neurol Neurosurg Psychiatry. 2002; 73:281-288. [PubMed: 12185159]

9. Ponsford J, Willmott C, Rothwell A, et al. Cognitive and behavioral outcome following mild traumatic head injury in children. J Head Trauma Rehabil. 1999; 14:360-372. [PubMed: 10407209]

10. Taylor HG, Dietrich A, Nuss K, et al. Post-concussive symptoms in children with mild traumatic brain injury. Neuropsychology. 2010; 24:148-159. [PubMed: 20230109]

11. Yeates KO, Luria J, Bartkowski H, et al. Postconcussive symptoms in children with mild closed head injuries. J Head Trauma Rehabil. 1999; 14:337-350. [PubMed: 10407207]

12. Hajek CA, Yeates KO, Gerry Taylor H, et al. Relationships among post-concussive symptoms and symptoms of PTSD in children following mild traumatic brain injury. Brain Inj. 2010; 24:100 109. [PubMed: 20085447]

13. Polissar NL, Fay GC, Jaffe KM, et al. Mild pediatric traumatic brain injury: adjusting significance levels for multiple comparisons. Brain Inj. 1994; 8:249-263. [PubMed: 8004083]

14. Hawley CA. Reported problems and their resolution following mild, moderate, and severe traumatic brain injury amongst children and adolescents in the U.K. Brain Inj. 2003; 17:105-129. [PubMed: 12519639]

15. Max, JE.; Ibrahim, F.; Levin, H. Neuropsychological and psychiatric outcomes of traumatic brain injury in children, in Cognitive and Behavioral Abnormalities of Pediatric Diseases. Nass, RD.; Frank, Y., editors. Oxford University Press; New York: 2010.

16. Max JE, Roberts MA, Koele SL, et al. Cognitive outcome in children and adolescents following severe traumatic brain injury: influence of psychosocial, psychiatric, and injury-related variables. J Int Neuropsychol Soc. 1999; 5:58-68. [PubMed: 9989025]

17. Babikian T, Satz P, Zaucha K, et al. The UCLA longitudinal study of neurocognitive outcomes following mild pediatric traumatic brain injury. J Int Neuropsychol Soc. 2011; 17:886-895. [PubMed: 21813031]

18. Carroll LJ, Cassidy JD, Peloso PM, et al. WHO Collaborating Centre Task Force on Mild Traumatic Brain Injury: Prognosis for Mild Traumatic Brain Injury: Results of the WHO Collaborating Centre Task Force on Mild Traumatic Brain Injury. J Rehabil Med. 2004; 43(Suppl): 84-105. [PubMed: 15083873]

19. Satz P, Zaucha K, McCleary C, et al. Mild head injury in children and adolescents: a review of studies (1970-1995). Psychol Bull. 1997; 122:107-131. [PubMed: 9283296]

20. Teasdale G, Jennett B. Assessment of coma and impaired consciousness: a practical scale. Lancet. 1974; 2:81-84. [PubMed: 4136544]

21. Levin HS, Hanten G, Roberson G, et al. Prediction of cognitive sequelae based on abnormal computed tomography findings in children following mild traumatic brain injury. J Neurosurg Pediatr. 2008; 1:461-470. [PubMed: 18518697]

22. Kaufman J, Birmaher B, Brent D, et al. Schedule for Affective Disorders and Schizophrenia for School-Age Children-Present and Lifetime Version (K-SADS-PL): initial reliability and validity data. J Am Acad Child Adolesc Psychiatry. 1997; 36:980-988. [PubMed: 9204677]

23. American Psychiatric Association. Diagnostic and Statistical Manual of Mental Disorders. 4th. American Psychiatric Press; Washington, DC: 1994.

24. Max JE, Castillo CS, Lindgren SD, et al. The Neuropsychiatric Rating Schedule: reliability and validity. J Am Acad Child Adolesc Psychiatry. 1998; 37:297-304. [PubMed: 9519635]

25. Boyle MH, Offord DR, Racine Y, et al. Identifying thresholds for classifying childhood psychiatric disorder: issues and prospects. J Am Acad Child Adolesc Psychiatry. 1996; 35:1440-1448. [PubMed: 8936910]

26. Leckman JF, Sholomskas D, Thompson WD, et al. Best estimate of lifetime psychiatric diagnosis: a methodological study. Arch Gen Psychiatry. 1982; 39:879-883. [PubMed: 7103676]

27. Association for the Advancement of Automotive Medicine. Abbreviated Injury Scale, 1990 Revision. Association for the Advancement of Automotive Medicine; Des Plaines, IL: 1990.

28. Andreasen NC, Endicott J, Spitzer RL, et al. The family history method using diagnostic criteria: reliability and validity. Arch Gen Psychiatry. 1977; 34:1229-1235. [PubMed: 911222] 
29. Miller IW, Epstein NB, Bishop DS, et al. The McMaster Family Assessment Device: reliability and validity. J Marital Fam Ther. 1985; 11:345-356.

30. Hollingshead, A. Four-Factor Index of Social Status. Yale University, Dept. of Sociology; New Haven, CT: 1975.

31. Sparrow, S.; Balla, D.; Cicchetti, D. The Vineland Adaptive Behavior Scales. American Guidance Services; Circle Pines, MN: 1984.

32. Woodcock, R.; Mather, N. Woodcock-Johnson Tests of Cognitive Ability. 3rd. DLM Teaching Resources; Allen, TX: 1989.

33. Yeates KO, Taylor HG. Predicting premorbid neuropsychological functioning following pediatric traumatic brain injury. J Clin Exp Neuropsychol. 1997; 19:825-837. [PubMed: 9524877]

34. Wechsler, D. Wechsler Intelligence Scale for Children. 3rd. The Psychological Corporation; New York: 1991.

35. Denckla MB, Rudel R. Rapid "automatized" naming of pictured objects, colors, letters, and numbers by normal children. Cortex. 1974; 10:186-202. [PubMed: 4844470]

36. Delis, D.; Kramer, J.; Kaplan, E., et al. CVLT-C: California Verbal Learning Test-Children's Version. The Psychological Corporation; San Antonio, TX: 1994.

37. Levin HS, Hanten G, Chang CC, et al. Working memory after traumatic brain injury in children. Ann Neurol. 2002; 52:82-88. [PubMed: 12112051]

38. Semel, E.; Wiig, EH.; Secord, WA. Clinical Evaluation of Language Fundamentals. 3rd. The Psychological Corporation; San Antonio, TX: 1995.

39. Cohen, J. Statistical Power Analysis for the Behavioral Sciences. Lawrence Erlbaum; Mahwah, NJ: 1988.

40. Max JE, Robin DA, Lindgren SD, et al. Traumatic brain injury in children and adolescents: psychiatric disorders at one year. J Neuropsychiatry Clin Neurosci. 1998; 10:290-297. [PubMed: 9706536]

41. Gerring JP, Brady KD, Chen A, et al. Premorbid prevalence of ADHD and development of secondary ADHD after closed head injury. J Am Acad Child Adolesc Psychiatry. 1998; 37:647654.

42. Max JE, Schachar RJ, Levin HS, et al. Predictors of secondary attention-deficit/hyperactivity disorder in children and adolescents 6 to 24 months after traumatic brain injury. J Am Acad Child Adolesc Psychiatry. 2005; 44:1041-1049. [PubMed: 16175109]

43. Max JE, Schachar RJ, Levin HS, et al. Predictors of attention-deficit/hyperactivity disorder within 6 months after pediatric traumatic brain injury. J Am Acad Child Adolesc Psychiatry. 2005; 44:1032-1040. [PubMed: 16175108]

44. Max JE, Castillo CS, Bokura H, et al. Oppositional defiant disorder symptomatology after traumatic brain injury: a prospective study. J Nerv Ment Dis. 1998; 186:325-332. [PubMed: 9653415]

45. Max JE, Levin HS, Landis J, et al. Predictors of personality change due to traumatic brain injury in children and adolescents in the first six months after injury. J Am Acad Child Adolesc Psychiatry. 2005; 44:434-442. [PubMed: 15843765]

46. Max JE, Levin HS, Schachar RJ, et al. Predictors of personality change due to traumatic brain injury in children and adolescents six to twenty-four months after injury. J Neuropsychiatry Clin Neurosci. 2006; 18:21-32. [PubMed: 16525067]

47. Anderson VA, Catroppa C, Haritou F, et al. Predictors of acute child and family outcome following traumatic brain injury in children. Pediatr Neurosurg. 2001; 34:138-148. [PubMed: 11359102]

48. Rivara JB, Jaffe KM, Fay GC, et al. Family functioning and injury severity as predictors of child functioning one year following traumatic brain injury. Arch Phys Med Rehabil. 1993; 74:10471055. [PubMed: 8215855]

49. Taylor HG, Yeates KO, Wade SL, et al. A prospective study of short- and long-term outcomes after traumatic brain injury in children: behavior and achievement. Neuropsychology. 2002; 16:15-27. [PubMed: 11853353]

50. Kesler SR, Adams HF, Blasey CM, et al. Premorbid intellectual functioning, education, and brain size in traumatic brain injury: an investigation of the cognitive reserve hypothesis. Appl Neuropsychol. 2003; 10:153-162. [PubMed: 12890641] 
51. Thurman D, Guerrero J. Trends in hospitalization associated with traumatic brain injury. JAMA. 1999; 282:954-957. [PubMed: 10485680] 


\section{TABLE 1}

Demographic, Psychosocial, and Injury Data on the Mild Traumatic Brain Injury Cohort (N=79)

\begin{tabular}{|c|c|c|}
\hline & & $\mathbf{N}$ \\
\hline \multicolumn{3}{|l|}{ Demographic Variables } \\
\hline Age at injury, years, mean (SD) & $9.81(2.93)$ & 79 \\
\hline Gender: boys, N (\%) & $54(68.4 \%)$ & 79 \\
\hline Socioeconomic status level, mean (SD) & $39.17(11.77)$ & 77 \\
\hline \multicolumn{3}{|l|}{ Psychosocial Variables } \\
\hline Pre-injury lifetime psychiatric disorder, $\mathrm{N}(\%)$ & $31(39.2 \%)$ & 79 \\
\hline Pre-injury Vineland Adaptive Behavior & $92.89(14.14)$ & 72 \\
\hline \multicolumn{3}{|l|}{ Composite Standard Score, mean (SD) } \\
\hline Pre-injury Family Functioning score, mean (SD) & $1.57(0.42)$ & 73 \\
\hline Family Psychiatric History score, mean (SD) & $1.10(1.04)$ & 63 \\
\hline Pre-injury Psychosocial Adversity, mean (SD) & $0.66(0.84)$ & 77 \\
\hline \multicolumn{3}{|l|}{ Injury Variables } \\
\hline Lowest Glasgow Coma Scale score: 13 & $6(7.6 \%)$ & \\
\hline Lowest Glasgow Coma Scale score: 14 & $18(22.8 \%)$ & \\
\hline Lowest Glasgow Coma Scale score: 15 & $55(69.6 \%)$ & \\
\hline Depressed skull fracture, N (\%) & $8(10.1)$ & 79 \\
\hline Mechanism of Injury & $\mathrm{N}(\%)$ & 79 \\
\hline Auto, truck, bus passenger & $17(21.5)$ & \\
\hline Recreational vehicle/off-road vehicle & $3(3.8)$ & \\
\hline Bicycle & $6(7.6)$ & \\
\hline Fall & $24(30.4)$ & \\
\hline Hit by a falling object & $3(3.8)$ & \\
\hline Sports or play & $10(12.7)$ & \\
\hline Hit by motor vehicle & $14(17.7)$ & \\
\hline Other & $2(2.5)$ & \\
\hline
\end{tabular}

J Neuropsychiatry Clin Neurosci. Author manuscript; available in PMC 2016 September 02. 
TABLE 2

Lesion Distribution, Based on Research Magnetic Resonance Imaging: Entire Cohort (N=65) and in Children With and Without Novel Psychiatric Disorder (NPD) in the First 12 Months After Injury

\begin{tabular}{|c|c|c|c|c|}
\hline & $\begin{array}{l}\text { All Subjects } \\
(\mathbf{N}=65)\end{array}$ & $\begin{array}{c}\text { NPD } \\
(\mathrm{N}=15) \\
\end{array}$ & $\begin{array}{l}\text { No NPD } \\
(\mathrm{N}=40) \\
\end{array}$ & \\
\hline & $\mathbf{N}(\%)$ & $\mathbf{N}(\%)$ & $\mathbf{N}(\%)$ & $\mathbf{p}$ \\
\hline Any lesion & $31(48)$ & $7(47)$ & $20(50)$ & NS \\
\hline Frontal lobe white matter & $7(11)$ & $2(13)$ & $3(8)$ & NS \\
\hline \multicolumn{5}{|l|}{ Distribution of other lesions } \\
\hline \multicolumn{5}{|l|}{ Frontal lobe } \\
\hline Any frontal lobe gray matter & $14(22)$ & $4(27)$ & $9(21)$ & \\
\hline Superior frontal gyrus & $7(11)$ & $2(13)$ & $5(13)$ & \\
\hline Middle frontal gyrus & $8(12)$ & $2(13)$ & $6(15)$ & \\
\hline Inferior frontal gyrus & $5(8)$ & $2(13)$ & $3(8)$ & \\
\hline Cingulate gyrus & $1(2)$ & 0 & $1(3)$ & \\
\hline Orbital gyrus & $1(2)$ & $1(7)$ & 0 & \\
\hline Gyrus rectus & $5(8)$ & $2(13)$ & $2(5)$ & \\
\hline Temporal lobe & $5(8)$ & $1(7)$ & $4(10)$ & \\
\hline Parietal lobe & $11(17)$ & $2(13)$ & $8(20)$ & \\
\hline Basal ganglia & $1(2)$ & $1(7)$ & 0 & \\
\hline Thalamus & $1(2)$ & 0 & $1(3)$ & \\
\hline
\end{tabular}


TABLE 3

Predictors of Novel Psychiatric Disorder (NPD) in the Interval 6-12 Months After Mild Traumatic Brain Injury (MTBI)

\begin{tabular}{|c|c|c|c|c|c|c|}
\hline & NPD $(\mathbf{N}=17)$ & No NPD $(\mathbf{N}=43)$ & $t$ & [df] & $\mathbf{p}$ & Effect Size \\
\hline \multicolumn{7}{|l|}{ Demographic Variables } \\
\hline Age at injury, years & $9.20(2.62)$ & $10.01(2.94)$ & 1.00 & [58] & NS & 0.29 \\
\hline Gender: boys $\mathrm{N}(\%)^{c}$ & $11(65 \%)$ & $29(67 \%)$ & & & NS & \\
\hline Socioeconomic Status & $33.25(11.40) \mathrm{N}=16$ & $40.56(11.86)$ & 2.13 & [57] & 0.038 & 0.63 \\
\hline Race $^{b}$ & & & & [4] & NS & \\
\hline Caucasian & 9 & 30 & & & & \\
\hline African-American & 1 & 6 & & & & \\
\hline Hispanic & 5 & 6 & & & & \\
\hline Asian & 2 & 0 & & & & \\
\hline Other & 0 & 1 & & & & \\
\hline \multicolumn{7}{|l|}{ Psychosocial Variables } \\
\hline Pre-injury lifetime psychiatric disorder, $\mathrm{N}(\%)^{\mathcal{C}}$ & $7(41 \%)$ & $16(37 \%)$ & & & NS & \\
\hline Pre-injury Vineland ABC Standard Score & $89.88(17.25) \mathrm{N}=16$ & $95.45(13.73) \mathrm{N}=40$ & 1.27 & [54] & NS & 0.36 \\
\hline Pre-injury Family Functioning & $1.77(0.41) \mathrm{N}=16$ & $1.53(0.43) \mathrm{N}=41$ & -1.96 & [55] & 0.055 & 0.57 \\
\hline Family Psychiatric History & $1.47(1.06) \mathrm{N}=15$ & $0.97(1.07) \mathrm{N}=37$ & -1.52 & [50] & NS & 0.47 \\
\hline Pre-injury Psychosocial Adversity & $1.13(1.02) \mathrm{N}=16$ & $0.60(0.82)$ & -2.02 & [57] & 0.048 & 0.57 \\
\hline \multicolumn{7}{|l|}{ Injury Variables } \\
\hline Lowest Glasgow Coma Scale Score (GCS) ${ }^{a}$ & & & & [2] & NS & \\
\hline Glasgow Coma Scale score: $13 ; \mathrm{N}$ & 2 & 3 & & & & \\
\hline Glasgow Coma Scale score: $14 ; \mathrm{N}$ & 5 & 11 & & & & \\
\hline Glasgow Coma Scale score: $15 ; \mathrm{N}$ & 10 & 29 & & & & \\
\hline Abnormal CT scan ${ }^{c}$ & $5 / 15(33 \%)$ & $16 / 41(39 \%)$ & & & NS & \\
\hline Depressed skull fracture $^{c}$ & $1 / 17(6 \%)$ & $6 / 43(14 \%)$ & & & NS & \\
\hline $\begin{array}{l}\text { Abbreviated Injury Scale: Injury Severity Score } \\
\text { (extracranial) }\end{array}$ & $2.7(6.9)$ & $1.3(2.7)$ & -0.80 & {$[15.6]$} & NS & 0.27 \\
\hline
\end{tabular}

Values are mean (standard deviation) except where indicated. Effect size refers to Cohen's d.

ABC: Adaptive Behavior Composite.

${ }^{a}$ Pearson $\chi^{2}=0.54$.

$b_{\text {Pearson }} \chi^{2}=8.25$.

$c_{\text {Fisher's exact test. }}$ 
TABLE 4

Neurocognitive Correlates of Novel Psychiatric Disorders (NPD) in the Interval 6-12 Months After Mild Traumatic Brain Injury (MTBI)

\begin{tabular}{|c|c|c|c|c|c|c|}
\hline & NPD $(\mathbf{N}=17)$ & No NPD $(\mathrm{N}=43)$ & $t$ & [df] & $\mathbf{p}$ & Effect Size \\
\hline \multicolumn{7}{|c|}{ Estimate of pre-injury academic functioning (conducted after the injury) } \\
\hline W-J-R Letter-Word ID standard score & $96.9(22.5)$ & $109.6(16.7) \mathrm{N}=42$ & 2.38 & [57] & 0.021 & 0.64 \\
\hline \multicolumn{7}{|c|}{ Academic and neurocognitive correlates 12 months post-injury } \\
\hline \multicolumn{7}{|l|}{ Academic functioning } \\
\hline W-J-R Letter-Word ID standard score & $96.2(22.0)$ & $109.5(16.4)$ & 2.57 & {$[58]$} & 0.013 & 0.69 \\
\hline \multicolumn{7}{|l|}{ Processing speed } \\
\hline WISC-III Processing Speed scale score & $104.0(19.5)$ & $112.9(17.9) \mathrm{N}=42$ & 1.68 & [57] & 0.099 & 0.48 \\
\hline Rapid Naming: z-score & $0.067(1.402)$ & $0.614(0.943)$ & 2.18 & {$[58]$} & 0.033 & 0.46 \\
\hline \multicolumn{7}{|l|}{ Memory functioning } \\
\hline CVLT: Monday list: long-delay z-score & $-0.21(1.40)$ & $0.69(0.81)$ & 3.09 & {$[58]$} & 0.003 & 0.79 \\
\hline N-back Letter Rhyme task hits & $0.31(0.27) \mathrm{N}=16$ & $0.51(0.23) \mathrm{N}=39$ & 2.86 & [53] & 0.006 & 0.80 \\
\hline \multicolumn{7}{|l|}{ Language } \\
\hline CELF-3 Formulated Sentences scaled score & $8.8(3.1)$ & $10.6(2.9) \mathrm{N}=42$ & 2.07 & {$[57]$} & 0.043 & 0.60 \\
\hline \multicolumn{7}{|l|}{ Adaptive Functioning 12 months post-injury } \\
\hline Vineland ABC Standard Score & $81.8(13.5)$ & $98.7(16.2) \mathrm{N}=42$ & 3.80 & {$[57]$} & 0.000 & 1.13 \\
\hline
\end{tabular}

Values are expressed as mean (standard deviation [SD]).

ABC: Adaptive Behavior Composite; CELF-3: Clinical Evaluation of Language Fundamentals, 3rd Edition; CVLT-C: California Verbal Learning Test, Children's Version; NS: not significant; p: significance level; WISC-III: Wechsler Intelligence Scale for Children, 3rd Edition; W-J-R: Woodcock-Johnson-Revised. 